\title{
Pelvic floor muscles training to reduce symptoms and signs of vulvovaginal atrophy: a case study
}

Mercier, Joanie PT, MSc; Morin, Mélanie PT, PhD; Lemieux, Marie-Claude MD; Reichetzer, Barbara MD, MSc; Khalifé, Samir MD; Dumoulin, Chantale PT, PhD

Funding/support: Regroupement Québécois de recherche sur le vieillissement, Ordre professionnel de la physiothérapie du Québec.

Financial disclosure/conflicts of interest: None reported.

\begin{abstract}
Objective: Vulvovaginal atrophy (VVA), caused by decreased levels of estrogen, is a common problem in aging women. Main symptoms of VVA are vaginal dryness and dyspareunia. First-line treatment consists of the application of local estrogen therapy (ET) or vaginal moisturizer. In some cases however, symptoms and signs persist despite those interventions. This case study describes a 77-year-old woman with severe VVA symptoms despite use of local ET and the addition of pelvic floor muscle (PFM) training to her treatment.

Methods: A patient with stress urinary incontinence and VVA was referred to a randomized clinical trial on PFM training. On pretreatment evaluation while on local ET, she showed VVA symptoms on the ICIQ Vaginal Symptoms questionnaire and the ICIQ-Female Sexual Matters associated with lower urinary tract Symptoms questionnaire, and also showed VVA signs during the physical and dynamometric evaluation of the PFM. She was treated with a 12-week PFM training program.

Results: The patient reported a reduction in vaginal dryness and dyspareunia symptoms, as well as a better quality of sexual life after 12 weeks of PFM training. On posttreatment physical evaluation, the PFMs' tone and elasticity were improved, although some other VVA signs remained unchanged.
\end{abstract}

Conclusions: Pelvic floor muscle training may improve some VVA symptoms and signs in women taking local ET. Further study is needed to investigate and confirm the present case findings and to explore mechanisms of action of this intervention for VVA. 
Vulvovaginal atrophy (VVA) is a common condition that affects $39 \%$ to $63 \%$ of postmenopausal women. 1 It results from decreased estrogen in the vulvovaginal tissues, 2 leading to blood flow reduction, 3 and the degeneration of collagen and elastin fibers,2-5 as well as $\mathrm{pH}$ and epithelium cell changes.2,6 These modifications cause a reduction of lubrication and sebaceous gland secretions, a loss of vulvovaginal tissue elasticity, and an environment more receptive to colonization by pathogenic bacteria.3,7 In patients with symptomatic VVA, an increase in pelvic floor muscle (PFM) tone can also be observed because of a protective guarding response triggered by pain and discomfort.8,9 VVA leads to vaginal dryness, pruritus, dyspareunia, urinary incontinence (UI), and increased frequency of vaginitis and urinary tract infection.1,2,7 All have negative impacts on general and sexual quality of life.1,10

Most common VVA treatments include local hormonal therapy (HT), systemic HT, application of vaginal moisturizer, and use of vaginal lubricants.3,11 According to the Society of Obstetricians and Gynaecologists of Canada and The North American Menopause Society, some of these treatments, however, may be not suitable for some patients, due to a variety of reasons (ie, risk of developing endometrial and breast cancer, allergies) and may be related to adverse effects (ie, vaginal irritation, vaginal bleeding, breast pain, nausea).3,7 In addition, according to a Cochrane systematic review, one study comparing estradiol ring treatment (a form of local HT) to placebo showed that $31 \%$ of women still felt vaginal dryness with this treatment.12 Dyspareunia also seemed to persist in some women treated with local estrogen therapy (ET) according to this Cochrane systematic review: in two studies comparing estrogen tablets to placebo, $20 \%$ to $31 \%$ of women still had dyspareunia.12 Therefore, there is a real need to investigate safe, effective, and low-cost complementary treatments.

PFM training refers to an exercise program, which specifically targets the PFM. It is usually taught and supervised by a health professional such as a physiotherapist.13 PFM training has proven to be an effective treatment for UI,14 genital prolapse,15-17 and dyspareunia.18,19 It is generally recommended as first-line treatment as it has been associated with low-cost and minimal adverse effects.14 PFM training could be beneficial for alleviating VVA symptoms and signs. Indeed, in a randomized control trial (RCT) investigating PFM training for UI, some women with VVA reported improvement of their VVA symptoms after the intervention. The case report below describes one of the participants in this RCT.

\section{CASE REPORT}

A 77-year-old multiparous woman was referred by her urogynecologist to the GROUP trial (Clinical trial.gov number NCT02039830). The objective of this noninferiority RCT was to compare the cost-effectiveness of group-based PFM training to individualized PFM training in the treatment of $\mathrm{UI}$ for women aged 60 and over. The PFM training program was the same in both groups; the only difference being its mode of delivery, that is either as group session or individual sessions. 
At pretreatment assessment, the participant complained of stress $\mathrm{UI}$ for 7 years and reported six urinary leakages per week mainly associated with coughing, walking, and effort as reported in a 1-week bladder diary. Other than UI, her actual general health was excellent. Her previous surgical history included abdominal hysterectomy without ovariectomy, cystocele repair, rectocele repair, and abdominal colposacropexy. She had been on oral ET for 10 years up to age of 70 . She was taking local ET (Premarin $0.625 \mathrm{mg} / \mathrm{g} \times 0.5 \mathrm{~g}$ ) once a week for 2 years.

This woman was sexually active, having intercourse with her husband (approximately three times per month). She had severe VVA symptoms, as measured by questionnaires related to urogynecological health (namely the ICIQ Vaginal Symptoms questionnaire [ICIQ-VS] and the ICIQ-Female Sexual Matters associated with lower urinary tract Symptoms questionnaire [ICIQFLUTSsex]). She reported her symptoms had begun 3 years ago.

The ICIQ-VS questionnaire assesses the severity of vaginal symptoms and associated sexual matters on quality of life using scales from "Never" to "All of the time" and from "Not at all" to "A lot." 20 On this questionnaire (see Table 1), the woman reported feeling vaginal dryness and vaginal tightness "All of the time." Both symptoms bothered her a lot $(8 / 10$ and $10 / 10$ on visual analogue scale [VAS], respectively). Furthermore, she expressed being worried "A lot" about VVA's impact on her sex life and feeling that her relationship with her partner was affected by vaginal symptoms "A little." Those declarations bothered her 10/10 and 5/10 on VAS, respectively. Finally, she reported that her sex life had been spoiled considerably by her vaginal symptoms (8/10 on a VAS) and that those symptoms interfered with her everyday life (4/10 on VAS).

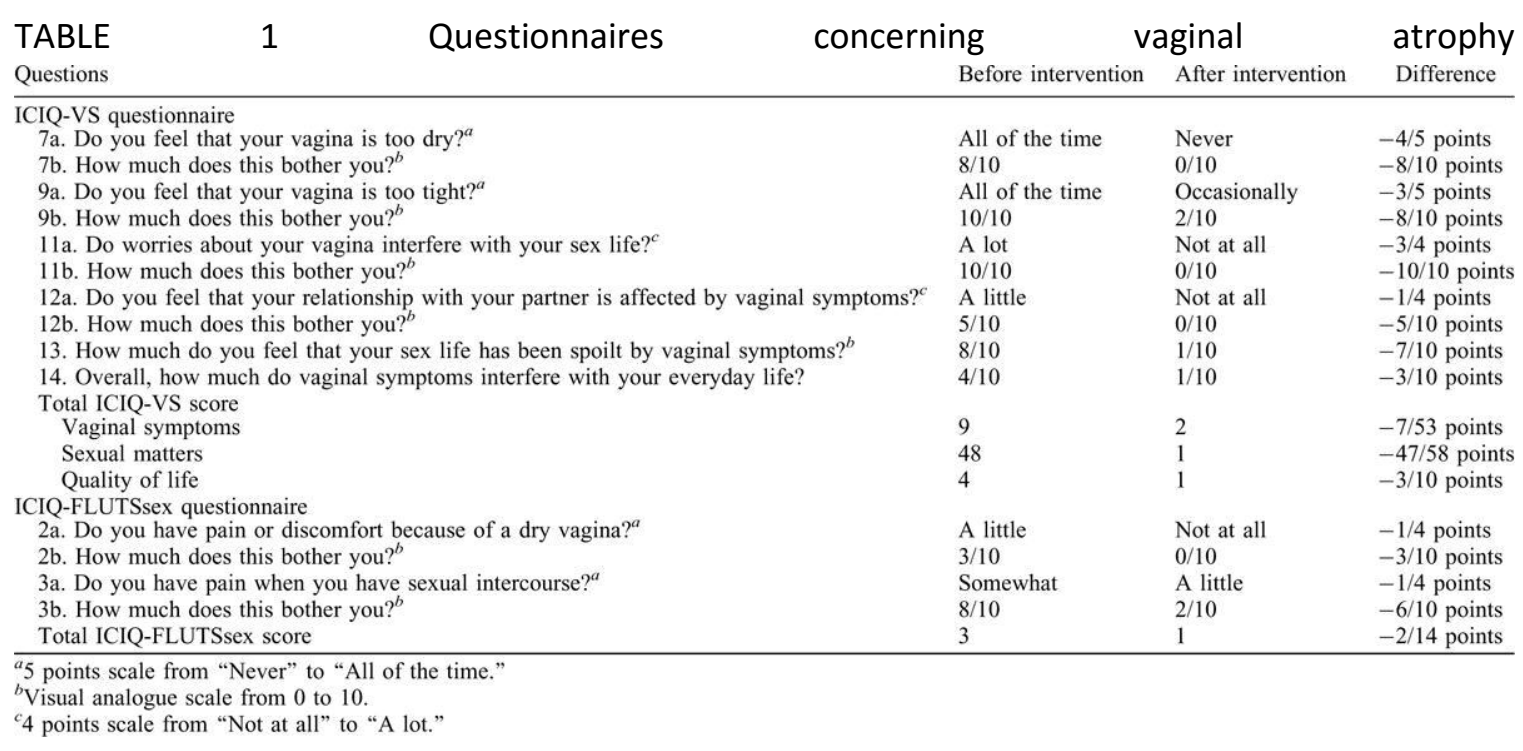

The ICIQ-FLUTS sex questionnaire values the sexual matters associated with lower urinary tract symptoms using scale from "Not at all" to "A lot."21 In this questionnaire (see Table 1), the 
woman complained of "A little" pain or discomfort because of a dry vagina and of "Somewhat" dyspareunia. Those symptoms bothered her at $3 / 10$ and at $8 / 10$ on VAS, respectively.

After completing the questionnaires, a physical therapy examination and a pelvic floor assessment were performed by an experienced physical therapist. More specifically, it included observations of the vulvovaginal area with the Vaginal Atrophy Index (VAI),22 PFM tone assessment with the Reissing scale, 8 and PFM tone and antero-posterior aperture assessment with a pelvic floor dynamometer 23 (see Table 2).

TABLE 2 Physical examination

\begin{tabular}{|c|c|c|c|}
\hline Assessment & Before intervention & After intervention & Difference \\
\hline \multicolumn{4}{|l|}{ Vaginal Atrophy Index } \\
\hline Skin elasticity & Poor & Fair & $-1 / 3$ points \\
\hline Pubic hair & Sparse & Sparse & 0 \\
\hline Labia & Atrophic, dry & Atrophic, dry & 0 \\
\hline Introitus & 2 fingerbreadths & 2 fingerbreadths & 0 \\
\hline Vaginal mucosa & Smooth & Smooth & 0 \\
\hline Vaginal depth & Normal & Normal & 0 \\
\hline Total & $10 / 15$ & $11 / 15$ & $-1 / 15$ points \\
\hline Reissing scale & +1 & 0 & -1 point \\
\hline \multicolumn{4}{|l|}{ Dynamometric assessment } \\
\hline Passive force (tone) & $1.42 \mathrm{~N}$ & $0.986 \mathrm{~N}$ & $-0.434 \mathrm{~N}$ \\
\hline Antero-posterior introital opening & $20.47 \mathrm{~mm}$ & $25.15 \mathrm{~mm}$ & $+4.68 \mathrm{~mm}$ \\
\hline
\end{tabular}

The VAI assesses the following six elements: vulvar skin elasticity, pubic hair, labia, vaginal mucosa, vaginal depth, and transverse introitus breadth, each on a scale from 1 to 3 or 1 to 2.22 Scores from $6 / 15$ to $11 / 15$ are related to more severe VVA; scores from $12 / 15$ to $14 / 15$ are related to slighter VVA; and a score of 15/15 represents no VVA according to Leiblum et al.22 In this index, we observed poor vulvar skin elasticity, sparse pubic hair, atrophic and dry labia, as well as smooth vaginal mucosa (absence of rugae) for this participant. The participant had normal vaginal depth and transverse introitus breadth (two fingerbreadths). Her total score on the VAI was 10/15 indicative of more severe VVA according to Leiblum scale.

Through vaginal digital palpation, the Reissing scale gives a subjective measure of PFM tone using a seven-point scale from -3 (hypotonic muscle) to +3 (hypertonic muscle) with normal value at 0.8 The participant showed some increased in PFM tone with a score of +1 on the right side, the left side, and the bottom.

The dynamometric speculum is an intravaginal dynamometer that can measure passive forces (tone) at different introital openings. At pretreatment assessment, the woman's passive force (tone) with the speculum branches closed to minimal aperture $(10 \mathrm{~mm})$ was $1.42 \mathrm{~N}$. When the dynamometer was open to maximal aperture, she had a somewhat reduced maximal anteroposterior introital aperture of $20.47 \mathrm{~mm}$ as compared with the mean opening for women of her age without VVA of $24.82 \mathrm{~mm}$ (unpublished data from our laboratory database).

After the pretreatment assessment, the participant was randomized in the group-based PFM training for an intensive physical therapy treatment, including weekly 1 hour PFM training supervised by another experienced physical therapist as described previously.24 Each session consisted of a 15-minute educational segment and a 45-minute exercise component. The 
educational segment covered PFM functions, different PFMs' problems, and women's health advices on UI (ie, liquid intake, constipation, and urgency control). The PFM exercise component included strength, endurance, and coordination exercises, as well as functional rehabilitation. More specifically, the participant did in each PFM training session maximal PFM contractions, fast PFM contractions, PFM contraction before and during a cough (the "knack" exercise), and an endurance exercise including a moderate PFM contraction, a maximal PFM contraction, and another PFM moderate contraction. Furthermore, PFMs were trained during functional activities.24 The treatment protocol was divided into three phases allowing for a gradual training progression, that is the gradual addition of increasingly difficult exercises in terms of duration, repetition, and position. Each phase lasted 4 weeks. The women had to perform the four PFM exercises included in the PFM training sessions at home, 5 days a week, for the duration of the treatment. To support training progression at home, the home-based exercise program was parallel to the three phases in the treatment protocol and also included gradual addition of increasingly difficult exercises every 4 weeks.

During the treatment phase ( $3 \mathrm{mo}$ ), the participant did her PFM exercises five times a week as recorded in her exercise diary for exercise compliance. She applied her local ET once a week during the treatment as she had done in the past 2 years.

After treatment, a posttreatment assessment was conducted. The woman showed a reduction of UI symptoms. On the 1-week bladder diary, there was a reduction in urinary leakage of $66.7 \%$, that is she had only two urinary leakages per week instead of six. The "minimal clinically important difference" for the bladder diary is considered to be a $50.0 \%$ reduction of urinary leakage which was attained.25

Improvement of some of her VVA symptoms and signs was also observed. On the ICIQ-VS questionnaire (see Table 1), she reported to "Never" feeling vaginal dryness anymore (reduction of $4 / 5$ points on the questionnaire scale) and only "Occasionally" vaginal tightness (reduction of $3 / 5$ points). Both symptoms bothered her less after the treatment $(0 / 10$ and $2 / 10$ on VAS respectively, both reductions of $8 / 10$ points). She reported she was not worried anymore about VVA's impacts on her sex life (reduction of 3/4 points) and VVA did not affect her relationship with her partner anymore (reduction of $1 / 4$ points). Therefore, she was not worried about those statements (both $0 / 10$ on VAS, reduction of $10 / 10$ points and 5/10 points, respectively). Finally, VVA did not affect her sex life as much as it did before, nor her overall quality of life, as she rated $1 / 10$ on VAS for both items (a reduction of $7 / 10$ points and $3 / 10$ points, respectively).

On the ICIQ-FLUTS sex questionnaire, the woman reported that she was not feeling any pain or discomfort because of a dry vagina anymore (reduction of $1 / 4$ points), and that it did not bother her anymore (reduction of $3 / 10$ points). She noted having "A little" dyspareunia (a reduction of $1 / 4$ points) that bothered her less (2/10 on VAS; a reduction of $6 / 10$ points).

On the posttreatment physical assessment, some improvements were observed, as well. Initially, on the VAI, only skin elasticity improved from "poor" to "fair." Sparse pubic hair, 
atrophic and dry labia, and smooth vaginal mucosa were still observed, as well as normal vaginal depth and normal transverse introitus breadth (two fingerbreadths). Therefore, the total score of the VAl improved by $1 / 15$ points, from $10 / 15$ to $11 / 15$. On the Reissing scale, the participant showed a normal tone with a score of 0 (a change of 1 point). These results corroborated with the posttreatment dynamometer assessment findings. Indeed, her passive force (tone) with the dynamometer close (10 mm opening) was decreased from 1.42 to $0.986 \mathrm{~N}$ (a reduction of 31\%). In addition, her maximal antero-posterior introital aperture increased from 20.47 to $25.15 \mathrm{~mm}$ (an increase of $23 \%$ ).

\section{DISCUSSION}

In regards to this 77-year-old woman, VVA caused significant symptoms and signs despite her following local ET. Symptoms of this pathology had negative impacts on her sex life and her everyday life. The participant also initially presented VVA signs of poor vulvar skin elasticity, sparse pubic hair, atrophic and dry labia, smooth vaginal mucosa, increased PFM passive force (tone), and decreased antero-posterior introital opening. After 3 months of PFM training, the woman appeared to have an important improvement in her VVA symptoms and signs.

The results of this case report are interesting and novel. To our knowledge, no other study investigated the effect of PFM training on VVA symptoms and signs. On the contrary, some studies looked at PFM training on global sexual function in incontinent women and had positive results.26-28 Liebergall et al 26 reported this global sexual function improvement after 12 weeks of PFM training in RCT (60 UI women), whereas Zahariou et al 27 and Beji et al 28 reported it in cohort studies after 12 months of PFM training (58 UI women) and 6 weeks of PFM training (42 UI women), respectively. Dyspareunia was also assessed and improved in those two cohort studies,27,28 but Liebergall's results were not statistically significant.26 The population of those studies was, however, different from the women with VVA population, as they were mainly younger premenopausal women. In Liebergall's study, mean age was 46.6 years old $(S D=8.9)$, and the authors did not give information about their menopausal status. In Zahariou's study, mean age was $43 \pm 6.2$ years old and $24.14 \%$ of participants were postmenopausal women. Finally, in Beji's study, mean age was $43.5 \pm 6.4$ years old and $26.2 \%$ of participants were postmenopausal women with ET.

The effects of PFM training were also evaluated in gynaecological cancer survivors with similar vaginal symptoms to women with VVA by Yang et al.29 Indeed, those women frequently feel less vaginal lubrication, less vaginal elasticity, and experience dyspareunia after being treated for their cancer.30 In Yang et al's RCT, this population aged from 35 to 67 years old significantly improved their sexual function and quality of life after 4 weeks of PFM training.29

The positive effects of PFM training on VVA that we have observed may be explained by different hypotheses. To begin with, PFM training could improve vulvovaginal blood flow. It is well recognized that skeletal muscle exercises contribute to increasing the number of capillaries in trained muscles.31 Because PFM arteries also supply the vulvovaginal tissues, PFM training 
could improve vulvovaginal blood flow and lead to a better vaginal lubrication as well as tissue quality. A similar hypothesis was introduced by Leiblum et al in an observational study with postmenopausal women in which sexually active women had significantly less overall VVA than sexually inactive women $(P=0.004) .22$ Increased blood flow to the pelvic organs by regular sexual activity would provide protection from VVA according to the author.22 It can be hypothesized that PFM training has an effect similar to intercourse on vulvovaginal blood flow.

In a second hypothesis, PFM training could improve elasticity of vulvovaginal tissues by repetitively mobilizing the different tissues' layers with repeated PFM contractions over time. Decreased estrogen at menopause makes collagen fibers fuse and undergo hyalinization, which leads to loss of mucosal elasticity as well as narrowing and shortening of the vagina.2 As shown in Madill et al with anatomical MRI, after 12 weeks of PFM training, a maximal PFM voluntary contraction lifts the urethrovesical junction approximately $7 \mathrm{~mm}$ (from $13.45 \pm 5.53 \mathrm{~mm}$ to $20.42 \pm 7.30 \mathrm{~mm}$ ).32 These tissues' mobilization could result in improved skin elasticity, an increased antero-posterior introital opening as well as a decreased feeling of vaginal tightness. This hypothesis can also explain why women with regular sexual activity have less VVA as vaginal penetration and stimulation also provokes vaginal tissues' mobilization.

Furthermore, the reduction of urinary leakage could lead to $\mathrm{pH}$ normalization. Zahariou et al elaborated this hypothesis in a cohort study including 12 months of PFM training.27 According to them, urinary leakages could modify vaginal $\mathrm{pH}$, affecting the normal flora and leading to vaginal dryness as well as loss of lubrication. 27 Reduction of urinary leakages would normalize vaginal $\mathrm{pH}$, leading to VVA symptoms' improvement.

Finally, PFM training could normalize PFM tone. The PFM exercises included in the 3-month program consist of repeated contractions and relaxations of PFM,14 which could have helped the women to be more aware of those muscles. Decreased PFM tone on posttreatment dynamometric assessment has also been shown by Madill et al 32 in a cohort study with incontinent women 60 years of age and over, after 12 weeks of PFM training $(P=0.047)$. Furthermore, Glazer et al in a cohort study of 33 women with provoked vestibulodynia after a 16-week biofeedback-assisted PFM training also noted a PFM resting tone decrease of $68 \%$, using electromyographic evaluation.33 So, PFM training seems to help women learn how to relax those muscles, which can be very useful during intercourse to reduce vulvovaginal tissues' frictions and dyspareunia.

The accidental discovery of potential VVA symptoms and signs improvement after PFM training in our noninferiority RCT is of high interest because of the simplicity, low cost, and absence of side effects of the intervention.14 It is also possible that these findings, however, may be related to a placebo effect or chance effect. A study with more robust methodology including a higher number of participants and targeting both VVA objective and subjective outcomes is needed to confirm the present case findings/results. 


\section{CONCLUSIONS}

The positive effects of PFM training in a postmenopausal woman with VVA have potential implications for treatments of VVA symptoms and signs. Further study is needed to investigate and confirm the present case findings and to explore mechanisms of action of this intervention on VVA.

\section{REFERENCES}

1. Parish SJ, Nappi RE, Krychman ML, et al. Impact of vulvovaginal health on postmenopausal women: a review of surveys on symptoms of vulvovaginal atrophy. Int J Womens Health 2013; 5:437-447.

2. Stika CS. Atrophic vaginitis. Dermatol Ther 2010; 23:514-522.

3. Johnston SL, Farrell SA, Bouchard C, et al. SOGC Joint Committee-Clinical Practice Gynaecology and UrogynaecologyThe detection and management of vaginal atrophy. J Obstet Gynaecol Can 2004; 26:503-515.

4. Weber MA, Limpens J, Roovers JPWR. Assessment of vaginal atrophy: a review. Int Urogynecol J 2015; 26:15-28.

5. Sturdee DW, Panay N. Recommendations for the management of postmenopausal vaginal atrophy. Climacteric 2010; 13:509-522.

6. Capewell AE, Mcintyre MA, Elton RA. Post-menopausal atrophy in elderly women: is a vaginal smear necessary for diagnosis? Age Ageing 1992; 21:117-120.

7. The North American Menopause SocietyManagement of symptomatic vulvovaginal atrophy: 2013 position statement of The North American Menopause Society. Menopause 2013; 20:888902.

8. Reissing ED, Brown C, Lord MJ, Binik YM, Khalifé S. Pelvic floor muscle functioning in women with vulvar vestibulitis syndrome. J Psychosom Obstet Gynecol 2005; 26:107-113.

9. Rosenbaum T. Managing postmenopausal dyspareunia: beyond hormone therapy. Female Patient 2006; 31:1-5.

10. Nappi RE, Kokot-Kierepa M. Vaginal Health: Insights, Views \& Attitudes (VIVA)-results from an international survey. Climacteric 2012; 15:36-44.

11. Woods NF. An overview of chronic vaginal atrophy and options for symptom management. Nurs Womens Health 2012; 16:482-494.

12. Suckling JA, Kennedy R, Lethaby A, Roberts $H$. Local oestrogen for vaginal atrophy in postmenopausal women. Cochrane Database Syst Rev 2006; 4:CD001500. 
13. Morris M, Randell M. Maternity and post-operative exercises in diagrams and words. JAMA 1937; 108:2161-2162.

14. Dumoulin C, Hay-Smith J, Habée-Séguin GM, Mercier J. Pelvic floor muscle training versus no treatment, or inactive control treatments, for urinary incontinence in women: a short version Cochrane systematic review with meta-analysis. Neurourol Urodyn 2015; 34:300-308.

15. Brækken IH, Majida M, Engh ME, B[circled division slash] K. Can pelvic floor muscle training reverse pelvic organ prolapse and reduce prolapse symptoms? An assessor-blinded, randomized, controlled trial. Am J Obstet Gynecol 2010; 203:170.e1-170.e7.

16. Hagen S, Stark D, Glazener C, Sinclair L, Ramsay I. A randomized controlled trial of pelvic floor muscle training for stages I and II pelvic organ prolapse. Int Urogynecol J Pelvic Floor Dysfunct 2009; 20:45-51.

17. Stüpp L, Resende APM, Oliveira E, Castro RA, Girão MJBC, Sartori MGF. Pelvic floor muscle training for treatment of pelvic organ prolapse: an assessor-blinded randomized controlled trial. Int Urogynecol J 2011; 22:1233-1239.

18. Goldfinger C, Pukall CF, Gentilcore-Saulnier E, McLean L, Chamberlain S. Original researchpain: a prospective study of pelvic floor physical therapy: pain and psychosexual outcomes in provoked vestibulodynia. J Sex Med 2009; 6:1955-1968.

19. Bergeron S, Brown C, Lord M-J, Oala M, Binik YM, Khalifé S. Physical therapy for vulvar vestibulitis syndrome: a retrospective study. J Sex Marital Ther 2002; 28:183-192.

20. Price N, Jackson SR, Avery K, Brookes ST, Abrams P. Development and psychometric evaluation of the ICIQ Vaginal Symptoms Questionnaire: the ICIQ-VS. BJOG 2006; 113:700-712.

21. Jackson S, Donovan J, Brookes S, Eckford S, Swithinbank L, Abrams P. The Bristol Female Lower Urinary Tract Symptoms questionnaire: development and psychometric testing. Br J Urol 1996; 77:805-812.

22. Leiblum S, Bachmann G, Kemmann E, Colburn D, Swartzman L. Vaginal atrophy in the postmenopausal woman: the importance of sexual activity and hormones. JAMA 1983; 249:2195-2198.

23. Dumoulin C, Bourbonnais D, Lemieux MC. Development of a dynamometer for measuring the isometric force of the pelvic floor musculature. Neurourol Urodyn 2003; 22:648-653.

24. Elliott V, de Bruin ED, Dumoulin C. Virtual reality rehabilitation as a treatment approach for older women with mixed urinary incontinence: a feasibility study. Neurourol Urodyn 2015; 34:236-243. 
25. Yalcin I, Peng G, Viktrup L, Bump RC. Reductions in stress urinary incontinence episodes: what is clinically important for women? Neurourol Urodyn 2010; 29:344-347.

26. Liebergall-Wischnitzer M, Paltiel O, Hochner Celnikier D, Lavy Y, Manor O, Woloski Wruble AC. Sexual function and quality of life of women with stress urinary incontinence: a randomized controlled trial comparing the Paula method (circular muscle exercises) to pelvic floor muscle training (PFMT) exercises. J Sex Med 2012; 9:1613-1623.

27. Zahariou A, Karamouti M, Papaioannou P. Pelvic floor muscle training improves sexual function of women with stress urinary incontinence. Int Urogynecol J 2008; 19:401-406.

28. Beji $\mathrm{N}$, Yalcin O, Erkan $\mathrm{H}$. The effect of pelvic floor training on sexual function of treated patients. Int Urogynecol J 2003; 14:234-238.

29. Yang EJ, Lim J-Y, Rah UW, Kim YB, et al. Effect of a pelvic floor muscle training program on gynecologic cancer survivors with pelvic floor dysfunction: a randomized controlled trial. Gynecol Oncol 2012; 125:705-711.

30. Bergmark K, Avall-Lundqvist E, Dickman PW, Henningsohn L, Steineck G. Vaginal changes and sexuality in women with a history of cervical cancer. N Engl J Med 1999; 340:1383-1389.

31. Egginton S. Invited review: activity-induced angiogenesis. Pflugers Arch 2009; 457:963-977.

32. Madill SJ, Pontbriand-Drolet S, Tang A, Dumoulin C. Effects of PFM rehabilitation on PFM function and morphology in older women. Neurourol Urodyn 2013; 32:1086-1095.

33. Glazer HI, Rodke G, Swencionis C, Hertz R, Young AW. Treatment of vulvar vestibulitis syndrome with electromyographic biofeedback of pelvic floor musculature. Obstet Gynecol Survey 1995; 50:658-659.

Keywords: Aging women; Pelvic floor muscles; Physical therapy; Vaginal atrophy

This is the accepted version of the following article: Mercier J, Morin M, Lemieux MC, Reichetzer B, Khalifé S, Dumoulin C. (2016).Pelvic floor muscles training to reduce symptoms and signs of vulvo-vaginal atrophy : a case study. Menopause. 2016 July; 23(7):816-820., which has been published in final form at

http://ovidsp.tx.ovid.com/sp-

3.21.1b/ovidweb.cgi?WebLinkFrameset=1\&S=PAMFFPBLNHDDLDPKNCIKGGMCLOCDAA00\&retu rnUrl=ovidweb.cgi\%3f\%26Complete\%2bReference\%3dS.sh.22\%257c1\%257c1\%26FORMAT\%3dci tationFormatPrint\%26FIELDS\%3dFORMATI\%26S\%3dPAMFFPBLNHDDLDPKNCIKGGMCLOCDAA00 \&directlink=http\%3a\%2f\%2fovidsp.tx.ovid.com\%2fovftpdfs\%2fFPDDNCMCGGPKNH00\%2ffs047 \%2fovft\%2flive\%2fgv031\%2f00042192\%2f00042192-201607000-

00018.pdf\&filename=Pelvic+floor+muscles+training+to+reduce+symptoms+and+signs+of+vulvo vaginal+atrophy\%3a++a+case+study.\&navigation links=NavLinks.S.sh.22.1\&PDFIdLinkField=\%2ff 
s047\%2fovft\%2flive\%2fgv031\%2f00042192\%2f00042192-201607000-

00018\&link from $=$ S.sh.22\%7c1\&pdf key=B\&pdf index $=S$. sh.22\&D=ovft 